\title{
La flexibilidad laboral en España y Perú: análisis de ciertos aspectos diferenciadores
}

\section{Labor flexibility in Spain and Peru: Analysis of some differential aspects}

\author{
SERGIO ARTURO QUIÑONES*
}

Resumen: En el presente artículo se efectúa un análisis comparado de los procesos de flexibilidad laboral llevados a cabo en las últimas décadas tanto en España como en Perú, buscando identificar los principales aspectos diferenciadores entre ambos a partir de tres factores: (i) la existencia o no de un marco político de referencia; (ii) las características del mercado de trabajo que inciden en la regulación de la contratación individual; y (iii) la potencialidad de la negociación colectiva.

Palabras clave: flexibilidad laboral - política de empleo - mercado de trabajo negociación colectiva

Summary: This paper contains a comparative analysis of the labor flexibility processes held in the past decades both in Spain and Peru, trying to identify the most relevant differential aspects between them based on the following three factors: (i) the existence —or not- of a political framework; (ii) the labor markets particularities that influence the regulation of the individual labor contracts; and (iii) the potential of unions and collective bargaining.

Keywords: labor flexibility - employment policies - labor market - collective bargaining

CONTENIDO: INTRODUCCIÓN.-- I. ANTECEDENTES.- II. ESTADO DE LA CUESTIÓN.-- II.1. ENFOQUES SOBRE LA FLEXIBILIDAD LABORAL.II.1.1. FLEXIBILIDAD COMO NECESIDAD IMPUESTA POR EL MERCADO.II.1.2. FLEXIBILIDAD COMO ESTRATEGIA EMPRESARIAL.- II.2. ALGUNOS APUNTES DESDE LA PERSPECTIVA JURÍDICA.- II.3. POSIBLES EFECTOS.III. EL CASO ESPAÑOL.- III.1. MARCO POLÍTICO DE REFERENCIA.- III.2. REGULACIÓN DE LA CONTRATACIÓN INDIVIDUAL.- III.3. POTENCIALIDAD DE LA NEGOCIACIÓN COLECTIVA.- IV. EL CASO PERUANO.- IV.1. MARCO POLÍTICO DE REFERENCIA.- IV.2. REGULACIÓN DE LA CONTRATACIÓN INDIVIDUAL.IV.3. POTENCIALIDAD DE LA NEGOCIACIÓN COLECTIVA.-V. CONCLUSIONES.

\footnotetext{
* Profesor de Derecho del Trabajo en la Pontificia Universidad Católica del Perú (PUCP). Abogado por la PUCP y magíster en Trabajo y Política Social por la Universidad Autónoma de Barcelona. Correo electrónico: sergio.quinones@ hotmail.com
} 


\section{NTRODUCCIÓN}

La flexibilidad laboral es el discurso dominante en materia de empleo en la actualidad; de hecho, lo ha sido durante las últimas tres décadas y no parece que vaya a dejar de serlo en los próximos años.

El discurso en cuestión ha sido extendido y asumido generalizadamente, aunque ha adoptado ciertos matices en cada Estado donde se ha implementado. Ello ha dependido de un conjunto de características propias, como son su cultura y tradición política, la estructura de su sistema productivo o la correlación de fuerzas entre sus actores sociales.

Precisamente, el objetivo del presente artículo es efectuar un análisis comparado de los procesos de flexibilidad laboral llevados a cabo en España y Perú, poniendo especial énfasis en identificar los aspectos diferenciadores entre ambos.

A manera de hipótesis, creemos que las principales diferencias se presentan básicamente debido a tres factores: (i) la existencia -en el caso español - de la Estrategia Europea de Empleo como marco político de referencia dentro del cual se inserta la flexibilidad, en tanto mecanismo para conseguir una serie de objetivos comunitarios; marco político que es inexistente en el caso peruano; (ii) aun cuando la marcada temporalidad de la contratación individual sea un elemento compartido, el mayor problema en el caso peruano sigue siendo la informalidad antes que la «dualidad» del mercado de trabajo, como es en el caso español; y (iii) la mayor potencialidad de la negociación colectiva en España frente al disminuido alcance que tiene en la actualidad en el Perú.

A tal efecto, iniciaremos nuestro análisis con unos breves apuntes - a manera de antecedentes - sobre el Estado de Bienestar para luego explorar los principales enfoques teóricos que abordan el concepto de flexibilidad laboral y sus posibles efectos. Así, sobre la base de un marco conceptual compartido, formularemos las principales diferencias que podamos identificar entre los procesos de flexibilización laboral de España y Perú, planteando, a su vez, una posible explicación para ello.

\section{ANTECEDENTES}

En el caso de Europa occidental, la flexibilidad laboral y el Estado de Bienestar son conceptos que se encuentran íntimamente vinculados. De hecho, como nos recuerda Recio ${ }^{1}$, el concepto de flexibilidad laboral fue puesto en circulación a inicios de la década de 1980 como un elemento clave para superar la crisis empresarial y de empleo imperante en la época, introduciendo así una noción que criticaba el modelo laboral productivo del Estado de Bienestar — fundamentado en el pensamiento

1 RECIO, Albert. «Aspectos económicos de la flexibilidad temporal». Trabajo, XIII (2004). 
keynesiano-, para proponer vías de solución a la crisis asociadas a la escuela neoclásica-liberal.

Efectivamente, las sociedades industriales de la posguerra se constituyeron tradicionalmente sobre la base del principio de estabilidad; vale decir, sobre la necesidad de eliminar la incertidumbre y el riesgo (de desempleo) a través de fuertes pactos sociales y regulaciones laborales que generaron empleo estable a los ciudadanos. Por su parte, en términos organizativos, este modelo se basó en el sistema de producción fordistataylorista: división del trabajo, organización jerárquica, producción en serie y mercado de masas.

En suma, como señalan Crespo y Serrano ${ }^{2}$, la estabilidad y el bienestar social se institucionalizaron mediante el establecimiento del Estado de Bienestar y la norma social del empleo estable. En este marco, el individuo era considerado miembro de una colectividad, en la que el empleo era la base de un sistema contributivo de seguridad social cuyos beneficios incluían, entre otros, la protección contra el desempleo, y esta protección era un derecho intrínseco a la condición de ciudadano.

En la misma línea, Miguélez ${ }^{3}$ apunta que el modelo de empleo en Europa occidental, luego de la Segunda Guerra Mundial, era el empleo estable, y precisa que, aunque no fue la situación de todos los ciudadanos — sobre todo de las mujeres_-, sí fue la norma a la que razonablemente se aspiraba como sociedad. De allí que las reformas estructurales que se introducen en la década de 1980 - como consecuencia de la crisis económica de la década anterior- empiezan a quebrar o, en ciertos países, a romper la norma social del Estado de Bienestar.

Ciertamente, con el cambio de modelo productivo, al de la «sociedad global del conocimiento», el riesgo es considerado algo inevitable, por lo que la flexibilidad — como idea de adaptación al cambio- resulta necesaria para afrontar dicha incertidumbre. Así, en vez de proteger a los individuos contra el riesgo, el Estado debería brindar las herramientas para que los individuos puedan manejar dicho riesgo. En definitiva, es el individuo el responsable directo de manejar el riesgo de desempleo, pues ya no se trata de un derecho adquirido, sino de una «ciudadanía» que hay que ganarse ${ }^{4}$.

Ahora bien, la secuencia de acción descrita en las líneas previas no necesariamente resulta aplicable al caso de América Latina, e incluso menos al de un Estado en concreto como el Perú. De hecho, tal como

2 CRESPO, Eduardo \& Amparo SERRANO. Are activation models converging in Europe? Bruselas: ETUI, 2004.

3 MIGUÉLEZ, Fausto. «La flexibilidad laboral». Trabajo, XIII (2004).

4 Crespo, Eduardo \& Amparo SerRano. Ob. cit.

LA FLEXIBILIDAD

LABORAL EN

ESPAÑA Y PERÚ:

ANÁLISIS DE

CIERTOS ASPECTOS

DIFERENCIADORES

LABOR FLEXIBILITY

IN SPAIN AND

PERU: ANALYSIS

OF SOME

DIFFERENTIAL

ASPECTS 
está definido, podemos afirmar que el Estado de Bienestar nunca se estableció en el Perú como modelo social.

La Organización Internacional del Trabajo (OIT), en un documento de análisis sobre las reformas laborales aplicadas en los países latinoamericanos, señala que luego de la crisis de la década de 1930 el modelo de desarrollo económico prevaleciente en América Latina fue el de la economía «cerrada» y el de la aplicación de una política de crecimiento «hacia adentro», cerrando su mercado a la competencia internacional, modelo que también fue denominado de «sustitución de importaciones».

En la mayoría de países este modelo mantuvo su vigencia por lo menos hasta la década de los ochenta, época en la que la crisis económica acentuó el cuestionamiento del «Estado Protector» y del modelo de desarrollo económico «cerrado», que se presumía agotado en la medida que daba muestras de incapacidad para mantener el crecimiento económico, generar empleos productivos, controlar la inflación y mejorar o, cuando menos, mantener el nivel de los ingresos ${ }^{5}$.

En este contexto, Brasil y Chile ensayaron modelos de inserción en una economía mundial o globalizada aplicando recetas más ortodoxas, basadas en la revalorización del papel del mercado en la asignación de recursos. El éxito particular del modelo chileno animó a que muchos países pusieran en ejecución programas de «ajuste estructural», entre los que podemos encontrar a Bolivia, Costa Rica y México, a partir de 1985, Colombia en 1990, Argentina en 1991 y Perú en 1992.

Parte de estos denominados «ajustes estructurales» supusieron, naturalmente, la flexibilidad del mercado laboral. En ese sentido, el propio documento de la OIT deja en evidencia que - junto con Argentina - en el Perú «las reformas han sido más profundas y han tenido un carácter marcadamente flexibilizador ${ }^{6}$.

En definitiva, sea como una crítica al Estado de Bienestar de Europa occidental -que supone un modelo no solo productivo, sino fundamentalmente social - o como un «efecto contagio» generado por la necesidad de modificar el modelo productivo de sustitución de importaciones en América Latina, la flexibilidad laboral se ha impuesto en España y Perú como el esquema indiscutible a seguir — hasta ahoraen materia de mercado de trabajo.

5 OIT. «Reformas laborales y procesos de integración en los países de la OEA: 1980-2000». Lima: Oficina Internacional del Trabajo, Documento de Trabajo 147, 2002.

6 Ibídem, p. 3. 


\section{ESTADO DE LA CUESTIÓN}

\section{II.1. Enfoques sobre la flexibilidad laboral}

Aunque se trata de un concepto que lleva ya tres décadas inmerso en el vocabulario académico y político, la flexibilidad no deja ser una noción ambigua. Como bien apunta Óscar Ermida, el uso del término en cuestión está teñido de intencionalidad, de una clara toma de posición ideológica. De este modo, se suele oponer la expresión «flexibilidad» como algo positivo frente a las condiciones negativas que supone su contraria, la «rigidez», que «evocaría calificativos de tosco, burdo, torpe, inadaptable»?

En la misma línea de razonamiento, Recio nos recuerda que la rigidez suele ser considerada una de las causas básicas del desempleo, utilizándose esta línea argumental para explicar los mayores niveles de desempleo que padecía la Unión Europea frente a otros países capitalistas desarrollados como Japón o Estados Unidos ${ }^{8}$.

En todo caso, lo cierto es que, en el ámbito internacional, la definición invocada con mayor frecuencia ha sido la consignada en el llamado «Informe Dahrendorf», preparado por un grupo de expertos de la Organización para la Cooperación y el Desarrollo Económico (OCDE), y que la concibe como la capacidad de los individuos en la economía y en particular en el mercado de trabajo de renunciar a sus costumbres y de adaptarse a nuevas circunstancias.

Pues bien, en las siguientes líneas analizaremos dos enfoques que buscan explicar las razones que han impulsado esta necesidad global de que los individuos y, en concreto, los mercados de trabajo sean flexibles, vale decir, tengan «capacidad de adaptación».

\section{II.1.1. Flexibilidad como necesidad impuesta por el mercado}

Cabría empezar señalando que este es el enfoque mayoritario y generalizado, tanto en la literatura especializada como en el discurso político. A partir de esta visión, la flexibilidad fue introducida, extendida y consolidada en los mercados de trabajo durante las últimas décadas.

La idea central, como explica Miguélez, es considerar a la «rigidez» del modelo de producción fordista-taylorista como la causa principal de la crisis de los años setenta y ochenta, en tanto le impedía adecuarse a las «nuevas demandas» del mercado. Se señalaba que la producción en grandes series se había estancado porque no había suficiente demanda en los países centrales — saturados ya de ese tipo de bienes_ y no se habían

7 OIT. «Estudios sobre la flexibilidad en el Perú». Lima: Oficina Internacional del Trabajo, Documento de Trabajo 124, 2000, p. 9.

8 RECIO, Albert. «Flexibilidad laboral y desempleo en España (reflexiones al filo de la reforma laboral)». Cuadernos de Relaciones Laborales, 5 (1994).

\section{LA FLEXIBILIDAD LABORAL EN ESPAÑA Y PERÚ: ANÁLISIS DE CIERTOS ASPECTOS DIFERENCIADORES}

LABOR FLEXIBILITY

IN SPAIN AND

PERU: ANALYSIS

OF SOME

DIFFERENTIAL

ASPECTS 
abierto nuevos mercados en otros países. En esa línea de pensamiento, se postulaba que se mantendría la demanda de quienes buscan «diferencia y distinción» y que siguen teniendo un gran poder adquisitivo. Por ello, la rígida organización del trabajo fordista-taylorista debía ser modificada hacia modos de producción más «a la carta», cambiantes y flexibles.

En suma, como señala el autor citado, «se dice que el mercado de bienes y servicios se ha flexibilizado en el sentido de que es menos estándar y se ha tenido que especializar según demandas más particulares, lo que ha llevado a la flexibilización — es decir, una utilización más diversificadade la tecnología y del trabajo y estas, necesariamente, a la del mercado de trabajo y del empleo»?

En igual sentido, Recio señala que - bajo el enfoque que venimos analizando— - la flexibilidad laboral supondría adaptación de las empresas al «nuevo» mercado y concesión de derechos y beneficios por parte de los trabajadores, pues se consideraba que el mayor obstáculo para la adaptación de las primeras era el exceso de protección de estos últimos. Ahora bien, este «nuevo» mercado se caracterizaría por la inestabilidad económica propia de la época de los ochenta (recurrentes shocks), la importancia del nuevo marco competitivo internacional y la existencia de nuevas y más variadas demandas de bienes y servicios.

Sin embargo, nos parece interesante reseñar algunos de los cuestionamientos que el mencionado autor formula a esta idea de un «nuevo» mercado: (i) la «volatilidad» de los mercados se asume como inherente al sistema, cuando no como resultado de una mala política económica; (ii) la competitividad internacional parece haberse reducido a la exigencia de menores derechos laborales y salarios a fin de abaratar precios; y (iii) la existencia de nuevas y variadas demandas puede ponerse en duda porque parecen más bien inducidas por una propaganda consumista, antes que generadas autónomamente, y porque no toman en cuenta la creciente desigualdad distributiva entre la población ${ }^{10}$.

Efectivamente, consideramos que el enfoque que hemos reseñado solo se puede sustentar en tanto la «idea-base» de un mercado global con una demanda de bienes y servicios cambiante y diversa que impone la flexibilización se mantenga «incólume»; lo que parece altamente discutible o, al menos, que así lo sea de manera generalizada, sin admitir particularidades por países, sistemas productivos y sectores económicos. 


\section{II.1.2. Flexibilidad como estrategia empresarial}

Desde este enfoque, la flexibilidad es, en realidad, una estrategia empresarial diseñada para enfrentar la incertidumbre del mercado y de la tecnología a través de un mayor control del factor trabajo.

Efectivamente, en línea con las críticas anotadas al final del acápite anterior, Miguélez señala que no siempre estamos ante la presencia de un mercado de bienes y servicios más flexible, en realidad, más que productos o servicios «a la carta», se trata de los mismos productos y servicios con «variaciones a la carta». En tal sentido, la flexibilización sería la respuesta a la construcción artificial de una realidad con el fin de justificar prácticas empresariales sobre el factor trabajo. Así, «el origen del proceso podría estar directamente en una estrategia de flexibilización del mercado de trabajo y del empleo con el ánimo de reducir los costes del trabajo o de introducir criterios más ágiles y más fáciles de control del mismo» ${ }^{11}$.

Con mayor razón, el citado autor recuerda que las empresas han tenido que conjugar siempre cuatro factores con el objetivo de generar ganancias: la tecnología, el mercado, el trabajo y el entorno político. Durante la época previa a la crisis de los ochenta, existía certeza para los empresarios sobre los cuatro factores: la tecnología era constante y duradera, el mercado estaba protegido, y el factor trabajo y el entorno político eran estables debido a la norma social del empleo en el Estado de Bienestar.

Sin embargo, con el fenómeno de la globalización económica se incrementan las incertidumbres respecto al factor tecnología y al factor mercado: los mercados se hacen inestables y la tecnología se hace obsoleta a gran velocidad. Dichas incertidumbres ya no pueden ser despejadas a nivel interno de cada país, ni siquiera con apoyo del Estado; por tanto, se rompen las reglas de equilibrio hasta entonces vigentes en muchos países, y los empresarios van variando su estrategia con el fin de controlar cada vez más en exclusiva el tercer factor, el trabajo. De allí que en opinión de Miguélez y Prieto, «ese es el significado profundo de la llamada flexibilización del trabajo [...] la utilización, según conveniencia, en extensión y en intensidad, del trabajo (jornada) y de la fuerza de trabajo (empleo) permite desplazar las incertidumbres del proceso hacia los trabajadores mismos» ${ }^{12}$.

Como puede apreciarse, desde este enfoque, la atención central no recae en un «nuevo mercado» que «impone» la flexibilidad, sino en unas «empresas» que «deciden» cambiar sus estrategias hacia la flexibilización.

LA FLEXIBILIDAD

LABORAL EN ESPAÑA Y PERÚ:

ANÁLISIS DE

CIERTOS ASPECTOS DIFERENCIADORES

LABOR FLEXIBILITY

IN SPAIN AND

PERU: ANALYSIS

OF SOME

DIFFERENTIAL ASPECTS 
En definitiva, para las empresas, flexibilidad supone tener certidumbre sobre el factor trabajo y más y mejor disponibilidad de su fuerza de trabajo; por el contrario, para los trabajadores significa incertidumbre de cara al futuro y cada vez menor disponibilidad sobre su vida cotidiana.

\section{II.2. Algunos apuntes desde la perspectiva jurídica}

Sea como necesidad impuesta por el mercado o como estrategia empresarial, la flexibilidad se ha traducido jurídicamente en un cambio de las normas que regulan el contrato de trabajo.

Estas nuevas reglas han incrementado la libertad del empleador, que originariamente había sido recortada por el Estado y los sindicatos - debido a la disparidad del poder de negociación de las partes-, permitiéndole tener ahora mayor control sobre el factor trabajo en un mundo en que el mercado y la tecnología son inciertos.

De este modo, desde la perspectiva jurídica, la flexibilidad laboral ha sido sinónimo de «desrregulación»; vale decir, no de eliminación de la norma laboral, sino de una nueva manera de regular el contrato de trabajo, que se aleja de la protección laboral clásica, con la finalidad — real o presunta - de aumentar la inversión, el empleo o la competitividad de la empresa.

Ciertamente, como señala Romagnoli, el concepto de «desrregulación» resume las orientaciones legislativas que convergen hacia un desenlace común: «ayudar a las empresas a salir, más competitivas que antes, del túnel de la crisis económica de los años setenta, restituyendo a las mismas los márgenes para una gestión flexible de la mano de obra suprimidos por el preexistente derecho sindical y del trabajo, ya fuese de origen legislativo o convencional ${ }^{13}$.

Ahora bien, en este acápite nos interesa llamar la atención sobre el hecho de que esta «desrregulación» laboral conduce, como lo hemos adelantado, a que el empresario traslade el «riesgo» (o incertidumbre) económico - inherente a la actividad productiva - hacia el trabajador, lo que, en nuestra opinión, desnaturaliza el fundamento de la relación laboral.

En efecto, debemos recordar que el contrato de trabajo es el vínculo jurídico entre un trabajador que pone su fuerza de trabajo a disposición de un empleador, quien le retribuye con un salario. En dicha puesta a disposición (subordinación) el trabajador le confiere al empleador el poder de dirigir su actividad. Es decir, el contenido de la promesa en el contrato de trabajo es uno de actividad subordinada, y no de resultado

13 RomAGNOLI, Umberto. «La desrregulación y las fuentes del derecho del trabajo». Cuadernos de Relaciones Laborales, I (1992), p. 15. 
(condicionado a la ganancia o pérdida), de allí que resulte esencial que el trabajador sea ajeno a los riesgos de la actividad empresarial.

Consideramos, pues, que la flexibilidad o «desrregulación» conduce - entre otros efectos perniciosos que veremos más adelante- a la desnaturalización del contrato de trabajo, toda vez que permite al empleador trasladar hacia el trabajador los riesgos (incertidumbres) de su actividad empresarial. Ello, desde la perspectiva jurídica, al menos, debería ser un factor ineludible de análisis al implementar medidas de flexibilización laboral.

\section{II.3. Posibles efectos}

Distintos autores coinciden en señalar que la flexibilidad laboral puede conducir a la precarización del empleo ${ }^{14}$. Ciertamente, aunque no son sinónimos, la aplicación de diversos tipos de flexibilidad —sobre todo la numérica externa e interna ${ }^{15}$ - puede conducir a la precarización del mercado laboral. De hecho, como se explicará más adelante, este ha sido uno de los efectos más perjudiciales generados por los procesos de flexibilización laboral implementados en España y Perú.

Y es que la precariedad no es solo temporalidad del empleo, se trata en realidad de llevar poco a poco a los trabajadores a una situación tal de inseguridad y vulnerabilidad que les impida incluso tomar el control de sus propios proyectos de vida. Comporta, pues, temporalidad, pero también falta de formación, bajos salarios, malas condiciones, jornadas discontinuas, incertidumbre, entre otros elementos.

En síntesis, la precariedad, como fenómeno laboral y social, «reduce la capacidad y autonomía de los trabajadores para planificar y controlar su vida profesional y social, a la vez que comporta una acusada asimetría de las relaciones de poder entre capital y trabajo que definen la relación laboral ${ }^{16}$.

Ahora bien, a lo anterior debe agregarse como uno de los principales efectos de la flexibilidad el de la «descolectivización» de la clase trabajadora.

Efectivamente, como señala Castel, con la crisis que se inició a mediados de la década de 1970, y que continua hasta la fecha, se produce la desagregación de la sociedad salarial, es decir, «la trayectoria ascendente de la consolidación del asalariado se rompió, lo que puso en cuestión la

14 Sobre el particular, revisar: CANO, Ernest. «La lógica de la precariedad laboral: el caso de la industria valenciana del mueble». Cuadernos de Relaciones Laborales, XIII (1998); MIGUÉLEZ, Fausto. Ob. cit.; y ALOS, Ramón. «Segmentación de los mercados de trabajo y relaciones laborales. El sindicalismo ante la acción colectiva». Cuadernos de Relaciones Laborales, XXVI, 1 (2008).

15 Ajuste a las fluctuaciones de la demanda del volumen de empleo y del volumen de horas de trabajo, respectivamente.

16 Cano, Ernest. Ob. cit., p. 208.

\section{LA FLEXIBILIDAD \\ LABORAL EN \\ ESPAÑA Y PERÚ: \\ ANÁLISIS DE \\ CIERTOS ASPECTOS \\ DIFERENCIADORES}

LABOR FLEXIBILITY

IN SPAIN AND

PERU: ANALYSIS

OF SOME

DIFFERENTIAL

ASPECTS 
asociación creciente del trabajo y de las protecciones que el progreso social parecía promover ${ }^{17}$.

Surgen, pues, con esta etapa dos grandes riesgos para los asalariados: el del paro y el de la precariedad; ambos riesgos quiebran las «homogeneidades intracategoriales» y rompen las «solidaridades intracategoriales que reposaban en la organización colectiva del trabajo y en la homogeneidad de situaciones compartidas por amplios colectivos de trabajadores» ${ }^{18}$.

En definitiva, en un mercado laboral flexible, mientras más precarias se vuelvan las condiciones del empleo, más obligados se verán los trabajadores a competir entre sía fin de proteger su empleo e irse «ganando la vida». Se genera así la «descolectivización» de los trabajadores y se pierde la noción de clase y la posibilidad de reivindicación colectiva de sus intereses.

Precarización del empleo y «descolectivización» de los trabajadores son, pues, en buena cuenta, los principales y más perjudiciales efectos que, en mayor o menor medida, pueden y se han generado como consecuencia de la flexibilidad laboral.

\section{EL CASO ESPAÑOL}

Como fue señalado en el apartado introductorio, en las páginas que siguen nos dedicaremos a analizar tres aspectos puntuales de los procesos de flexibilización llevados a cabo en España y Perú, empezando por España: (i) la existencia o no de un marco político de referencia; (ii) la regulación de la contratación individual; y (iii) la potencialidad de la negociación colectiva.

En el caso español, como se detallará a continuación, observamos que no se ha producido un único proceso de flexibilización laboral que marque un antes y un después en su mercado de trabajo. Por el contrario, entendemos que la flexibilidad ha sido introducida en España a través de una serie de reformas laborales sucedidas en las últimas décadas, y no todas necesariamente en el mismo sentido.

De hecho, en el mes de septiembre de 2010 entró en vigencia la ley 35/2010 que estableció medidas urgentes para la reforma del mercado de trabajo, ello con el objetivo esencial de contribuir a la reducción del desempleo e incrementar la productividad de la economía española.

Más aun, estas primeras semanas del año 2012 vienen siendo escenario de un intenso diálogo social entre el flamante gobierno del Partido

17 CASTEL, Robert. «¿Por qué la clase obrera ha perdido la partida?» En DíAZ-SALAZAR, Rafael (dir.). Trabajadores precarios. Los proletarios del siglo XXI. Madrid: HOAC, 2003, p. 174.

18 Ibídem, p. 176. 
Popular, las organizaciones sindicales y las empresariales en torno a la implementación de una nueva y profunda reforma laboral que enfrente el agudo problema del desempleo derivado de la crisis financiera europea. El proceso de flexibilización laboral en España, pues, parece estar lejos de haber concluido.

En tal sentido, corresponde en este punto advertir que el análisis que se presenta en los apartados siguientes se basa en un marco institucional y normativo que podría ser objeto de importantes modificaciones en las próximas semanas o meses del año en curso ${ }^{19}$.

\section{III.1. Marco político de referencia}

Desde la entrada en vigencia del Tratado de Roma, a fines de la década de 1950, el pleno empleo siempre ha sido uno de los objetivos constantes de la Unión Europea (UE). La explicación la encontramos en la íntima vinculación que se le atribuye a la cohesión social y al pleno empleo.

Ciertamente, como señalan Miguélez y Prieto, «al menos desde el Tratado de Maastricht, en 1987, la cohesión económica y social se ha convertido para las autoridades de la UE en uno de los objetivos políticos más relevantes» ${ }^{20}$, y aunque la causalidad entre ambos conceptos es bastante compleja, se entiende que en la mayoría de los países que hoy componen la UE, la cohesión social pudo ser lograda como resultado de una base sólida de bienestar individual y de políticas sociales que tuvieron como eje la existencia de pleno empleo. Así, se puede afirmar que para la UE el fortalecimiento de la cohesión económica y social pasa por facilitar el acceso al empleo.

Ahora bien, esta preocupación por el empleo a la que hemos aludido, que se remonta a los orígenes mismos de la UE, se consolida en 1997 con la aprobación de la Estrategia Europea de Empleo (EEE) ${ }^{21}$.

En efecto, a raíz de la introducción del nuevo título VIII sobre el Empleo en el Tratado de Ámsterdam de 1997, el Consejo Europeo adoptó la EEE en la cumbre extraordinaria sobre el empleo celebrada en Luxemburgo en noviembre de 1997, a fin de coordinar las políticas nacionales en dicha materia.

La EEE crea un marco de vigilancia multilateral y se dirige, en particular, a que los Estados miembros se comprometan sobre un

19 Efectivamente, durante la semana en que culminó la redacción del presente artículo se publicó en el Boletín Oficial del Estado el Real Decreto Ley 3/2012, de 10 de febrero, de Medidas Urgentes para la Reforma del Mercado Laboral. Los efectos que esta nueva reforma laboral producirá en el mercado de trabajo español y, de modo particular, en la dinámica de sus relaciones individuales y colectivas de trabajo, es un tema que amerita un próximo estudio complementario.

20 MiguéleZ, Fausto \& Carlos PRIETO. Ob. cit., p. 225.

21 Las referencias en torno a la EEE tienen como fuente la web oficial de la UE (http://europa.eu/ legislation_summaries/employment_and_social_policy/index_es.htm).

LA FLEXIBILIDAD

LABORAL EN

ESPAÑA Y PERÚ:

ANÁLISIS DE

CIERTOS ASPECTOS

DIFERENCIADORES

LABOR FLEXIBILITY

IN SPAIN AND

PERU: ANALYSIS

OF SOME

DIFFERENTIAL

ASPECTS 
conjunto de objetivos y metas comunes en torno a cuatro pilares: (i) la «empleabilidad», como mecanismo de lucha contra el desempleo de larga duración y el desempleo de los jóvenes; (ii) el fomento del espíritu empresarial, a través de normas claras, estables y fiables para la creación y la gestión de empresas y la simplificación de las obligaciones administrativas para las pequeñas y medianas empresas; (iii) la adaptabilidad de las empresas a través de la modernización de la organización y la flexibilidad del trabajo; y (iv) la igualdad de oportunidades entre hombres y mujeres en el mercado de trabajo.

Como puede apreciarse, la flexibilidad laboral (pilar III) se inserta como un mecanismo más para conseguir una serie de objetivos comunitarios mayores. En ese sentido, Martínez señala que

todo indica que la inserción por lo económico y el empleo serían la piedra angular del actual modelo de política social europea. Siendo esto así, la EEE sería el eje central sobre el que gira la política social europea, y las medidas sociales que se diseñan y proponen para la integración socio laboral de los individuos ${ }^{22}$.

Siguiendo a Hyman ${ }^{23}$, podemos afirmar que, pese a que la integración europea ha sido primordialmente un proyecto de construcción de un mercado común que ha eludido la creación de una política social y de empleo con carácter vinculante, la EEE termina constituyendo un marco político de referencia que establece pilares y objetivos alineados con el modelo social europeo al que se aspira.

A ello debe agregarse el que el Método Abierto de Coordinación - metodología de trabajo introducida por la EEE - facilite el debate político a distintos niveles y mediante un enfoque integrado: las acciones adoptadas en el ámbito del empleo han de ser coherentes con los ámbitos cercanos al empleo, como las políticas sociales, la educación, el régimen fiscal, la política empresarial y el desarrollo regional.

En definitiva, creemos que la existencia de la EEE significa un elemento diferenciador del proceso de flexibilidad español, pues tiene la potencialidad de permitir a los actores sociales, políticos, comunidades académicas y ciudadanía en general, analizar, debatir, evaluar y criticar la flexibilidad laboral dentro de un marco político mayor de referencia; y no como medidas en sí mismas, aisladas y desconectadas de un proyecto nacional macro, como consideramos sucede en el Perú.

22 MARTínEZ, Antonio. «El discurso de la Unión Europea en materia de políticas de empleo y exclusión social. Análisis sociológico de la Estrategia Europea de Empleo». Papers, XCVI, 1 (2011), p. 47.

23 HYMAN, Richard. «La rigidez flexible: ¿un modelo para una Europa social?». Cuadernos de Relaciones Laborales, XIX (2001). 


\section{III.2. Regulación de la contratación individual}

En línea con diversos autores que hemos consultado ${ }^{24}$, tenemos que la regulación de la contratación individual en España ha pasado por una interesante evolución histórica que se inicia con cierta jurisprudencia y ordenanzas laborales — previas a 1976 - que elaboran el principio de causalidad: las empresas deben contratar a trabajadores indefinidos para sus actividades permanentes y constantes, la contratación a plazo definido debe justificarse en causas temporales.

Este principio es consagrado legalmente en la Ley de Relaciones Laborales (LRL) de 1976, regulando tres contratos de duración determinada: obra o servicio, eventual e interinidad. El resto de contrataciones laborales debían ser indefinidas, con excepción de un conjunto de decretos dictados entre 1976 y 1979 que permitió utilizar el contrato temporal como política de lucha contra el desempleo.

El Estatuto de los Trabajadores de 1980 apuesta por un modelo dual de contratación temporal: una estructural (manteniendo los contratos de la LRL de 1976) y una coyuntural (a través de la autorización al Gobierno para implantar contratos temporales de fomento del empleo).

Un hito importante lo constituye la reforma laboral de 1984, pues con ella se flexibilizan los contratos temporales estructurales, al crear un cuarto tipo (lanzamiento de nueva actividad) y, sobre todo, al consolidar legalmente la contratación temporal de fomento del empleo. De este modo, en la década 1984-1994 se margina la modalidad contractual indefinida y se inicia un imparable proceso de precarización del mercado laboral español.

Ciertamente, como señala Recio, en esta época el mercado laboral español se convirtió en el más flexible de los países de la UE en materia de contratación laboral, con un crecimiento espectacular de empleo temporal: la tasa de temporalidad se incrementó del 15,8\% en 1987 al $32,6 \%$ en $1993^{25}$.

Ahora bien, con la reforma laboral de 1994 comienza la restauración del principio de causalidad en los contratos temporales, pese a que el Gobierno sigue haciendo uso de la contratación temporal como medida de fomento del empleo y se legalizan las empresas de trabajo temporal.

La reforma laboral de 1997 refuerza el principio de causalidad al derogar el contrato de lanzamiento de nueva actividad y eliminar las políticas

24 AGUILERA, Raquel. «El principio de «causalidad» en la contratación laboral». Revista del Ministerio de Trabajo y Asuntos Sociales, XXXIII (2001); LAHERA, Jesús \& Juan Carlos GARCíA. "La transformación de la contratación laboral, el trabajo a tiempo parcial y el despido». Cuadernos de Relaciones Laborales, XXV, 2 (2007); y RECIO, Albert. «Flexibilidad laboral y desempleo en España (reflexiones al filo de la reforma laboral)"

25 Ibídem, p. 62.

LA FLEXIBILIDAD

LABORAL EN

ESPAÑA Y PERÚ:

ANÁLISIS DE

CIERTOS ASPECTOS

DIFERENCIADORES

LABOR FLEXIBILITY

IN SPAIN AND

PERU: ANALYSIS

OF SOME

DIFFERENTIAL

ASPECTS 
de incentivo general del empleo temporal. Por el contrario, se crea un contrato de fomento de la contratación indefinida con un menor coste de despido improcedente y al que se confieren bonificaciones en el pago de las cuotas de Seguridad Social, destinado a algunos colectivos. Pese a ello, la alta tasa de temporalidad en España nunca llega a bajar del 31\%.

Entre el año 2001 y 2005 se consolida el contrato de fomento de la contratación indefinida para amplios colectivos, pero se crea el contrato temporal de inserción, destinado al sector público y a las entidades sin ánimo de lucro, contrato que es finalmente derogado en la reforma laboral de 2006, la que, a su vez, introduce un novedoso límite en el encadenamiento de contratos temporales con un mismo trabajador en la misma empresa. A pesar de esta medida, y de las renovadas subvenciones al empleo fijo, la tasa de temporalidad hacia el 2007 se mantenía en un 34\%.

Finalmente, como fue señalado en párrafos anteriores, a fines de 2010 la ley 35/2010 introdujo medidas urgentes para la reforma del mercado de trabajo destinadas a reducir la dualidad del mercado de trabajo español, dentro de las que se incluye el establecimiento de un límite temporal máximo para los contratos de obra y servicio, se mejora la regla para evitar el encadenamiento de contratos temporales y se incrementa el monto de la indemnización por finalización de los contratos temporales.

Habiendo efectuado esta breve síntesis de la evolución histórica de la regulación del contrato individual de trabajo, podemos concluir que, aun cuando el marco normativo vigente no contiene un contrato normalizado de fomento del empleo temporal (por el contrario, hay una lista cerrada y tasada de contratos temporales), sí crea un escenario propicio para la generación de este tipo de empleo, lo que explicaría la continuidad a lo largo de los años de la alta tasa de temporalidad en España, que, según datos de la Encuesta de Población Activa, se ubicó en el 25,4\% en el año 2009.

En opinión de Lahera y García $^{26}$, este «escenario propicio» para la contratación temporal se genera por las siguiente razones: (i) el amplio ámbito de los contratos temporales permite la contratación de trabajadores temporales en numerosos supuestos; (ii) la regulación flexible de la contratación temporal —en materia de protecciones y formalidades al contrato mismo-; (iii) la indemnización no es un factor disuasivo por su bajo coste; y (iv) la inexistencia de costes al encadenamiento de contratos temporales - hasta la reforma laboral de 2006-. 
Antes de concluir con el presente acápite, consideramos relevante llamar la atención sobre un aspecto en particular: la informalidad laboral. Como bien apunta Voge ${ }^{27}$, no existe ningún dato preciso que permita hacer comparaciones en el área europea, pero la realidad es que el «trabajo clandestino» afecta a categorías muy diversas de trabajadores, siendo los más vulnerables los trabajadores inmigrantes que no poseen permiso de residencia o de trabajo; en otros casos afecta a trabajadores en situación de desempleo, independientes, mujeres que no tienen acceso al mercado regulado de trabajo ni al paro, entre otros.

Sobre la base de la literatura e información estadística revisada, entendemos que la informalidad laboral y el impacto que ella genera en la composición del mercado de trabajo no ha sido considerada en los estudios sobre la flexibilidad laboral en España, lo que constituye un segundo aspecto diferenciador con el caso peruano.

\section{III.3. Potencialidad de la negociación colectiva}

Una primera característica del modelo sindical español es la elevada concentración de la afiliación, tanto sindical como empresarial, en un número reducido de organizaciones de representación colectiva que actúan a nivel nacional. En el seno de estas asociaciones se concentran las decisiones de actividad sindical, siendo sus cúspides de dirección quienes marcan las líneas más relevantes en materia de estrategia de negociación de los convenios ${ }^{28}$.

Una segunda característica que menciona Cruz Villalón es la amplia experiencia desarrollada en la celebración de diversos Acuerdos Marco (Acuerdo Básico de Empleo, Acuerdo Marco Interconfederal, Acuerdo Nacional de Empleo, Acuerdo Interconfederal, Acuerdo Económico y Social), a través de los cuales se concertaba entre las partes las innovaciones principales a introducir en los contenidos de los convenios colectivos en todo el territorio nacional.

Ello se debe a que el Estatuto de los Trabajadores, desde su primitiva redacción de 1980, admite la celebración de Convenios Marco, de ámbito estatal o de Comunidad Autónoma, para regular determinados aspectos estructurales de la negociación colectiva, con el fin de articular en cierta medida las negociaciones entre diferentes unidades de contratación en sus respectivos ámbitos de vigencia o establecer las reglas de solución de los conflictos entre convenios. Instaura, pues, una vía de centralización y articulación negocial ${ }^{29}$.

27 VoGEL, Laurent. «Derecho del trabajo y precarización de derechos en los Estados de la Unión Europea». Cuadernos de Relaciones Laborales, IX (1996).

28 CRUZ VILLALÓN, Jesús. «Estructura y concurrencia entre convenios colectivos». Revista del Ministerio de Trabajo y Asuntos Sociales, LXVIII (2007).

29 GalianA, Jesús. «NEGOCIACIÓN COLECTIVA». Revista del Ministerio de Trabajo y Asuntos Sociales, LVIII (2005).

LA FLEXIBILIDAD

LABORAL EN

ESPAÑA Y PERÚ:

ANÁLISIS DE

CIERTOS ASPECTOS

DIFERENCIADORES

LABOR FLEXIBILITY

IN SPAIN AND

PERU: ANALYSIS

OF SOME

DIFFERENTIAL

ASPECTS 
Como resultado de ello, tenemos que desde el año 1997 se han suscrito distintos Acuerdos Interconfederales para la Negociación Colectiva de nivel estatal, los cuales, con mayor o menor éxito, establecieron criterios generales, orientaciones y recomendaciones para la negociación de los convenios colectivos, tanto en materia salarial, como en diferentes aspectos de las relaciones laborales.

Finalmente, cabe destacar como una característica adicional del modelo sindical español el que, aunque el grado de densidad sindical (porcentaje de trabajadores asalariados afiliados a los sindicatos) es bastante reducido, el nivel de cobertura de la negociación colectiva (porcentaje de trabajadores potencialmente sujetos a la negociación colectiva cubiertos por la misma) es relativamente elevado en comparación con otros países de la UE.

Así, a manera de ejemplo, se señala que aunque la tasa de afiliación en España subió solo de 9\% en 1980 a 15\% en 2000 — de las más bajas en la UE_, el nivel de cobertura de la negociación colectiva es de casi 80-85\%, ello debido a la eficacia general automática de los convenios colectivos prevista por ley, independientemente de que los trabajadores estén o no afiliados a los sindicatos firmantes ${ }^{30}$.

Sobre el particular, la Encuesta de Calidad de Vida en el Trabajo 2009 establece que el porcentaje de ocupados afiliados a algún sindicato en dicho año se ha elevado al 17,2\%; asimismo, según el Anuario de Estadísticas del Ministerio de Trabajo e Inmigración, en el año 2009 se celebraron un total de 5.002 convenios colectivos - entre nuevos y revisados - comprendiendo en sus alcances a 10.160.170 trabajadores.

En suma, se trata, pues, de un modelo de relaciones colectivas fuertemente centralizado, con vasta experiencia en el diálogo social y, aunque tiene una baja tasa de afiliación sindical —en comparación con la UE-, cuenta con un amplio nivel de cobertura de la negociación colectiva.

\section{EL CASO PERUANO}

Siguiendo a Javier Neves ${ }^{31}$, podemos afirmar que el ordenamiento laboral peruano moderno ha tenido solo dos sistemas de relaciones laborales: (i) el de la primera fase del régimen militar a inicios de la década de 1970; y (ii) el instaurado como consecuencia de la reforma laboral llevada a cabo a inicios de la década de 1990. 
Como señala el citado autor, pese a que dichos sistemas tuvieron orientaciones opuestas, se puede constatar en ambos dos características comunes: la metodología autoritaria de su puesta en práctica y la desconfianza por las organizaciones sindicales autónomas. Ello supuso que ambos sistemas fueran impuestos desde el Estado sin diálogo ni concertación con el interlocutor sindical (pese a que sí lo hubo con el empresarial).

Ahora bien, tanto Neves ${ }^{32}$ como Villavicencio ${ }^{33}$ coinciden en apuntar que la necesidad de sustituir el sistema laboral del régimen militar se planteó desde dos perspectivas distintas: la jurídica y la económica. La primera se fundamentaba en la entrada en vigencia de la Constitución de 1979, que adoptaba un modelo de estado democrático y social, opuesto a los rasgos controlistas y represivos de la regulación militar. En el plano económico, América Latina sufría también los efectos de la crisis económica de la década de 1980, lo que impulsaba la necesidad de flexibilizar los mercados laborales, a lo que se sumaba la introducción de innovaciones tecnológicas a las que era necesario adecuarse.

Se trataba, pues, «de disminuir el exceso de regulación estatal de detalle, dejando márgenes mayores de decisión a la autonomía privada, especialmente en el plano colectivo, pero también en el individual [...] De este modo se daría una adecuación apropiada a las nuevas circunstancias» ${ }^{34}$.

Ahora bien, es importante resaltar que en el caso peruano la reforma laboral de los noventa generó, primero, una desrregulación de las relaciones individuales de trabajo, haciendo retroceder al Estado en su función compensadora, para luego regular intensamente las relaciones colectivas, manteniendo el esquema intervencionista y restrictivo histórico. Así, la desrregulación en lo individual (reforma) y la «sobrerregulación» en lo colectivo (continuismo) resultaron caminos complementarios e intensamente vinculados a un objetivo común: fortalecer la posición del empresario en el establecimiento de las condiciones de trabajo ${ }^{35}$.

En definitiva, y como se detalla en las siguientes páginas, el ajuste estructural de los noventa en el Perú buscó favorecer al máximo la posición empresarial, en tanto política a través de la cual se generarían más y mejores empleos, lo que — ahora queda en evidencia— nunca

33 VILLAVICENCIO, Alfredo. «El cuestionable continuismo intervencionista o la ausencia de reforma en la regulación general de las relaciones colectivas de trabajo y de la libertad sindical». En SOCIEDAD Peruana de Derecho del Trabajo y de la Seguridad Social (SpdtSS). Balance de la Reforma Laboral Peruana, Lima: La Sociedad, 2001.

34 NeVES, Javier. Ob. cit., p. 3.

35 VILLAVICENCIO, Alfredo. Ob. cit.

\section{LA FLEXIBILIDAD \\ LABORAL EN \\ ESPAÑA Y PERÚ: \\ ANÁLISIS DE \\ CIERTOS ASPECTOS \\ DIFERENCIADORES}

LABOR FLEXIBILITY

IN SPAIN AND

PERU: ANALYSIS

OF SOME

DIFFERENTIAL

ASPECTS 
sucedió. Todo lo contrario, la rebaja de los niveles de protección de los trabajadores y la casi desaparición de los sujetos colectivos fueron las consecuencias directas de la reforma laboral de los noventa.

\section{IV.1. Marco político de referencia}

Desde nuestro punto de vista, en este aspecto se pueden identificar de plano dos diferencias básicas con el caso español: (i) no existe en el Perú un marco político de referencia similar a la EEE; y (ii) al inicio de la década de 1990 el Perú sufrió una ruptura democrática ${ }^{36}$ que condujo a la aprobación de una nueva Constitución Política.

Efectivamente, con relación a lo primero, más allá de planes coyunturales a favor del empleo, usualmente cambiantes con cada gobierno de turno, no existe en el Perú un marco político de referencia que establezca pilares y objetivos marco que se alineen hacia la consecución de un modelo social nacional.

En nuestra opinión, dicha ausencia impide que la flexibilidad laboral sea objeto de un debate político y social serio y que se inserte como una medida coherente con otras políticas de índole social, educativa, fiscal y empresarial. Por el contrario, nuestra percepción es que las medidas de flexibilidad laboral implementadas en el Perú han sido concebidas como fines en sí mismas, aisladas y desconectadas de un proyecto nacional marco.

Como acertadamente anota Villavicencio, «parte de nuestro drama es que no somos capaces aún de generar un consenso social que se exprese en la Constitución, de manera que luego todos nos preocupemos, en mayor o menor medida, de exigir su cumplimiento. Y ese proceso, desgraciadamente, todavía continua inconcluso en el Perú» ${ }^{37}$.

Ahora bien, con relación a la segunda diferencia mencionada, es importante señalar que a inicios de la década de los noventa la Constitución Política de 1979 —adoptada luego del gobierno militar de los setenta - fue sistemáticamente «acusada» desde la tribuna oficialista y empresarial de ser extremadamente rígida en muchas de sus postulaciones. Desde allí se impulsó la necesidad de superar dichas rigideces y reglamentaciones excesivas que supuestamente venían retrasando el normal desarrollo evolutivo de la legislación laboral de nuestro país (hacia la flexibilidad, se entiende).

En este contexto, como señala Carrillo, la Constitución Política de 1993 se adopta — desde la perspectiva del empleo— con el objetivo de

36 El «autogolpe» de Estado perpetrado por el entonces presidente, Alberto Fujimori, el 5 de abril de 1992.

37 VilLAVICENCIO, Alfredo. Ob. cit., p. 275. 
permitir que se legisle a favor de la flexibilización del derecho laboral, incentivando con ello las inversiones privadas, facilitando el crecimiento económico y, supuestamente, la creación de más y mejores empleos. La tendencia marcadamente flexibilizadora que se adoptó no hace más que poner en evidencia que lo que buscó la «desconstitucionalización» de los derechos laborales fue «que el legislador ordinario o acaso el gobernante - con medidas extraordinarias- sea el libre y no condicionado configurador del nuevo derecho laboral ${ }^{38}$.

Con este nuevo marco constitucional, el proceso de flexibilización laboral llevado a cabo en el Perú en la década de los noventa no fue concertado ni negociado. En realidad, «se llevó a efecto fundamentalmente bajo la orientación de los empleadores, sin que los sindicatos puedan insertarse dentro del proceso, y se efectuó a través de decretos inconsultos por parte del Gobierno» ${ }^{39}$.

Efectivamente, como recuerda Ciudad, la política laboral del Gobierno en la década de 1990 se caracterizó por la ausencia total de mecanismos de diálogo social y concertación con los actores sociales. De hecho, las relaciones laborales, impregnadas de un clima de mutua desconfianza, no eran más que el reflejo de la ruptura democrática que se vivió desde 1992. En suma «durante la década del noventa no se valoró debidamente que el marco normativo de una sociedad pluralista debe suponer un compromiso, un pacto normativo de convivencia política entre los distintos sectores sociales ${ }^{40}$.

\section{IV.2. Regulación de la contratación individual}

$\mathrm{Al}$ igual que en el caso español, queremos empezar este acápite con una breve referencia a la evolución de la normativa que ha regulado la contratación individual en el Perú.

Sobre el particular, Neves ${ }^{41}$ nos recuerda que el primer sistema (el del gobierno militar de los setenta) giró en torno a dos instituciones fundamentales en materia de relaciones individuales de trabajo: (i) la estabilidad laboral, tanto en la contratación —-dando preferencia casi exclusiva a la contratación indefinida sobre la temporal-, como en la extinción — prohibiendo el despido injustificado—; (ii) la participación de los trabajadores en la propiedad, la gestión y las utilidades de las empresas.

38 CARRILLO, Martín. «La flexibilización del contenido laboral de la Constitución». En SPDTSS. Ob. cit., P. 56.

39 CIUDAD, Adolfo. «Efectos de la flexibilización del derecho individual del trabajo sobre las relaciones colectivas en el Perú». En SpDTSS. Ob. cit., p. 249.

40 lbídem, p. 251.

41 NeVES, Javier. Ob. cit.

LA FLEXIBILIDAD

LABORAL EN

ESPAÑA Y PERÚ:

ANÁLISIS DE

CIERTOS ASPECTOS

DIFERENCIADORES

LABOR FLEXIBILITY

IN SPAIN AND

PERU: ANALYSIS

OF SOME

DIFFERENTIAL

ASPECTS 
Ambas instituciones constituyeron la columna vertebral del régimen laboral durante la etapa militar, en tanto le proporcionaban «al trabajador la seguridad del mantenimiento de una relación duradera y pretendían comprometerlo con los resultados de la gestión empresarial, buscando sustituir la lógica del conflicto por la de la conciliación $»^{42}$.

A diferencia del anterior, el sistema laboral instaurado con la reforma de los noventa sí abordó la integridad de las instituciones que conforman las relaciones individuales de trabajo, y en todos los casos - como apunta el citado autor - resulta evidente la rebaja de beneficios para los trabajadores y el incremento de los márgenes de decisión empresarial (tal vez con la sola excepción de la compensación por tiempo de servicios).

En efecto, la reforma de los noventa atravesó por tres etapas (1991, 1995 y 1997), y cada una de ellas fue menos protectora que la anterior. El resultado de esta reforma es que «hoy una empresa tiene, en el Perú, muchas posibilidades de contratar personal que no esté a su cargo, o que estándolo no tenga garantías de permanencia en el empleo, ni perciba siquiera algunos beneficios indispensables ${ }^{43}$.

De este modo, el Estado ha cedido en su función compensadora de la desigualdad material entre trabajadores y empleadores, dejando un amplio margen de negociación que, lamentablemente, no ha sido llenado por la autonomía colectiva, sino por la individual, esto es, por la imposición unilateral de la voluntad empresarial.

En suma, se puede concluir que el principal efecto generado por la reforma laboral de los noventa ha sido la precarización del empleo,

hay una marcada disminución de la protección del trabajador, tanto en perspectiva horizontal (la población abarcada es menor, por el crecimiento de la informalidad, pero también por la admisión de modalidades no laborales de contratación de personal), como vertical (los derechos de esa población están severamente disminuidos, en calidad y cantidad $)^{44}$.

Ciertamente, otros autores ${ }^{45}$ también coinciden en señalar como el principal efecto generado por el proceso de flexibilización implementado en el Perú a la precarización del empleo; vale decir, que el ajuste del mercado de trabajo durante los noventa se centró en el incremento cuantitativo del empleo (precario), antes que en la mejora de la calidad del empleo. 
En la misma línea, Campana y Prelle concluyen que los resultados en materia de flexibilidad de la contratación laboral distan de ser satisfactorios. No solo tuvieron limitados efectos sobre la generación de empleo de calidad, sino que tuvieron negativas consecuencias sobre el funcionamiento del mercado de trabajo. Así, «el resultado práctico de las reformas en materia de contratación tiene que ver directamente con la precarización de las relaciones laborales, como con la sustitución de trabajadores estables por temporales (en aplicación combinada de la flexibilidad de entrada y de salida) e incluso clandestinos ${ }^{46}$.

Lo anterior se puede corroborar con información estadística ${ }^{47}$, de la que se desprende que a nivel nacional la tasa de asalariados privados contratados temporalmente se incrementó del 15,3\% en 1998 al 25,2\% en 2009. En cambio, la tasa de contratados a plazo indefinido se redujo del 17,6\% en 1998 al 11,2\% en 2009.

Esta dualidad del mercado laboral formal conlleva, además de que solo haya aumentado el empleo temporal en desmedro del permanente, un conjunto de efectos negativos adicionales que ha sido acertadamente puesto en evidencia por Cuadros y Sánchez ${ }^{48}$ : (i) se observa una importante brecha de ingresos entre trabajadores que pertenecen a un mismo grupo ocupacional pero que han sido contratados bajo distintas modalidades - la diferencia es de casi el triple, en promedio-; (ii) se podría deducir que las empresas usan intensivamente la contratación temporal como un elemento disuasorio contra la afiliación sindical, toda vez que la prerrogativa de renovación de contratos recae en los empleadores; y (iii) el uso intensivo de la contratación temporal incrementa la tasa de rotación laboral, lo cual ha redundado, por un lado, en una menor duración media del empleo —entre 1986 y 2006 se redujo de ocho a cuatro años en Lima Metropolitana- y, del otro, en un nivel pobre de capacitación laboral.

Pues bien, hasta este punto, los efectos de la flexibilidad laboral sobre la regulación de la contratación individual parecen ser similares en España y Perú: una marcada dualidad del mercado laboral que conduce a la precarización del empleo.

Sin embargo, como se advierte ya en la propia información estadística mostrada, en el caso peruano ha jugado un papel gravitante la elevadísima tasa de informalidad laboral y el impacto que ella genera

46 OIT. Ob. cit., p. 67.

47 INSTITUTO NACIONAL DE ESTADística E INFORMÁtICA (INEI). «Encuesta Nacional de Hogares, III Trimestre, 1998-2000»; «Encuesta Nacional de Hogares sobre Condiciones de Vida y Pobreza, continua, 2004-2008». Elaboración: MTPE-Programa de Estadísticas y Estudios Laborales (PEEL). Encuestas disponibles en el sitio web official del INEI (http://www.inei.gob.pe/).

48 CuADROS, Fernando \& SÁNCHEZ Christian. «La contratación temporal en el Perú: la informalidad escondida". Asesoría Laboral, diciembre (2007).

LA FLEXIBILIDAD

LABORAL EN

ESPAÑA Y PERÚ:

ANÁLISIS DE

CIERTOS ASPECTOS

DIFERENCIADORES

LABOR FLEXIBILITY

IN SPAIN AND

PERU: ANALYSIS

OF SOME

DIFFERENTIAL

ASPECTS 
en la composición del mercado de trabajo: $64 \%$ de informalidad en promedio durante el período revisado ${ }^{49}$.

En efecto,

el peso específico del empleo formal en la economía peruana sigue siendo muy pequeño. De acuerdo a estimaciones mediante las últimas encuestas nacionales de hogares, solo uno de cada cuatro empleos en la economía peruana sería considerado mínimamente formal (es decir, que cuenta efectivamente con por lo menos uno de los beneficios estipulados por la legislación laboral, tales como seguro de salud, vacaciones, CTS, gratificaciones, sistema de pensión, sindicalización o contrato permanente) ${ }^{50}$.

En la misma línea, se ha verificado que los índices de informalidad y sub empleo son alarmantes —entre los más altos de la región-y, pese a los esfuerzos que se realizan, no se aprecia una mejora en el mediano plazo. De hecho, el régimen común para los trabajadores peruanos es la informalidad («sin contrato»), es decir, la mayoría de personas son contratadas en forma verbal, bajo mecanismos informales o formales simulados ${ }^{51}$.

Precisamente, en atención a esta lucha contra la informalidad laboral, se aprobó en el año 2008 un régimen laboral especial para la micro empresa (hasta 10 trabajadores y un cierto tope en su nivel de ventas) y la pequeña empresa (hasta 100 trabajadores y un cierto tope en su nivel de ventas). Este régimen creó un estatuto diferenciado para los trabajadores empleados en dichas empresas, que supone - a grandes rasgos- la reducción generalizada de los derechos y beneficios laborales que tiene un trabajador sujeto al estatuto común.

Lo alarmante de ello es que, lejos de reducir los niveles de informalidad laboral, dada la estructura empresarial del mercado peruano, se ha sujetado aproximadamente al $76 \%$ del total de los asalariados privados a nivel nacional ${ }^{52}$ a un régimen profundamente desrregulador y reductor de derechos.

En conclusión, consideramos que en el caso peruano la «lucha» contra la informalidad supone la segmentación de derechos laborales por tamaño

49 INEI. «Encuesta Nacional de Hogares, III Trimestre, 1998-2000» y «Encuesta Nacional de Hogares sobre Condiciones de Vida y Pobreza, continua, 2004-2008». Elaboración: MTPE - Programa de Estadísticas y Estudios Laborales (PEEL). Encuestas disponibles en el sitio web official del INEI (http://www.inei.gob.pe/).

50 YAMADA, Gustavo. "Hacia una mayor flexibilidad y seguridad en el mercado laboral peruano". Economía y Sociedad, 70 (2008), p. 48.

51 TOYAMA, Jorge \& otros. "Retos pendientes para implementar la flexiguridad en el Perú». Documento de trabajo no publicado, preparado por encargo de la CEPAL, 2008.

52 INEI. «Encuesta Nacional de Hogares sobre Condiciones de Vida y Pobreza, continua, 2009». Elaboración: MTPE - Programa de Estadísticas y Estudios Laborales (PEEL). Encuesta disponible en el sitio web official del INEI (http://www.inei.gob.pe/). 
de empresa (diferentes estatutos de trabajador según seas de una micro, pequeña o mediana/gran empresa), lo que, en nuestra opinión, equivale a legalizar la precarización del empleo de la abrumadora mayoría de asalariados peruanos.

\section{IV.3. Potencialidad de la negociación colectiva}

En materia de relaciones colectivas de trabajo, el sistema del régimen militar tuvo un marcado sesgo de control sobre las organizaciones sindicales, tanto en su constitución como en su accionar. Afirma Neves que

solo podía existir un único sindicato por ámbito (unicidad sindical) lo que favorecía el poder sindical, pero contrariaba el Convenio 87 de la OIT. La autonomía estaba amenazada constantemente por diversos organismos del Estado que pretendían dotar al régimen de una presencia sindical. No olvidemos que había una central creada y fomentada por el régimen (la Central de Trabajadores de la Revolución Peruana) ${ }^{53}$.

En líneas generales, nos recuerda el citado autor, la negociación colectiva durante esta etapa estuvo marcada por un fuerte intervencionismo del Estado, quien participaba en cada una de las etapas por las que el procedimiento de negociación debía transcurrir.

Pese a esta injerencia en la autonomía sindical, los beneficios de los convenios colectivos eran de duración permanente por lo que la negociación colectiva tenía una tendencia progresiva que siempre incrementaba beneficios. Asimismo, aun cuando el nivel de negociación favorecido por el Estado era el de la empresa, no se llegó a impedir negociaciones a nivel de rama de actividad o gremio —en los que el poder sindical era mayor-.

La reforma de los noventa introdujo una nueva Ley de Relaciones Colectivas de Trabajo que, en opinión de Neves, constituyó una mejora en tres aspectos centrales: (i) disminuyó la injerencia del Estado sobre las negociaciones colectivas; (ii) permitió la pluralidad sindical; y (iii) abrió diversas vías para la solución de los conflictos colectivos, como son la conciliación, la mediación y el arbitraje.

No obstante ello, estas mejoras no se extendieron a aspectos centrales de la materia, por lo que resultaron insuficientes para consolidar a los sindicatos frente al empresariado, cuyo poder se había reforzado enormemente en el campo de las relaciones individuales de trabajo.

En efecto, hay cuatro aspectos clave por los que la reforma de los noventa debilita la potencialidad de la negociación colectiva:

LA FLEXIBILIDAD

LABORAL EN

ESPAÑA Y PERÚ:

ANÁLISIS DE

CIERTOS ASPECTOS

DIFERENCIADORES

LABOR FLEXIBILITY

IN SPAIN AND

PERU: ANALYSIS

OF SOME

DIFFERENTIAL

ASPECTS 
(i) adopta un modelo primitivo de fuero sindical — solo protege a ciertos dirigentes y solo frente al despido-; (ii) orienta compulsivamente la negociación colectiva al nivel de empresa, eliminando prácticamente los otros niveles de negociación ${ }^{54}$; (iii) establece una vigencia temporal de los beneficios pactados en los convenios colectivos; y (iv) asume un modelo estático de huelga — solo para fines profesionales y supone el cese total de labores con abandono del centro de trabajo- ${ }^{55}$.

Si a los aspectos legales antes mencionados se suma el hecho de que la reforma laboral en el aspecto individual condujo a la precarización del empleo, y esta generó una situación de extrema debilidad en la base misma de la vida sindical, tendremos la explicación de la grave crisis sindical presente al día de hoy en el Perú.

Ciertamente, como señalan Cuadros y Sánchez, luego de la reforma laboral de inicios de la década de 1990, las tasas de sindicación y la cantidad de convenios colectivos registrados sufrieron una drástica caída: en Lima Metropolitana, por ejemplo, el porcentaje de trabajadores sindicalizados se redujo del $21,9 \%$ en 1990 al 4,2\% en 2007; mientras que el número de convenios colectivos registrados a nivel nacional disminuyó de, aproximadamente, 2000 en 1990 a poco más de 500 en $2009^{56}$.

Entre las principales razones que explicarían esta disminución en la tasa de sindicación se encuentran el límite mínimo de trabajadores impuesto para constituir un sindicato (20 trabajadores para sindicato de empresa y 50 trabajadores para sindicatos de otra naturaleza), el uso intensivo de la contratación temporal por parte de la mediana y gran empresa, el crecimiento de la participación en el empleo de los trabajadores ocupados en el segmento de la microempresa, el incremento de la informalidad laboral, así como el debilitamiento de la capacidad fiscalizadora de la Autoridad Administrativa del Trabajo, entre otras ${ }^{57}$.

Para finalizar, queremos llamar la atención sobre una vital diferencia frente al caso español. Debido al diseño legal de nuestro modelo sindical, las bajas tasas de afiliación se traducen en bajas tasas de cobertura de la negociación colectiva ${ }^{58}$, lo que claramente disminuye la potencialidad de la negociación colectiva en el Perú.

54 De hecho, ha tenido que ser a través de sendas sentencias del Tribunal Constitucional que se recuperó el nivel de negociación colectiva a nivel de rama de actividad en la construcción y en los puertos.

55 NEVES, Javier. Ob. cit.

56 CUADROS, Fernando \& Christian SÁNCHEZ. «El rol estatal de fomento de la negociación colectiva». En Libro del III Congreso Nacional de la SPDTSS, 2008.

57 Ibídem.

$58 \mathrm{Al} 2007$ solo el 8,9\% de asalariados privados se encontraba sujeto a negociación colectiva (Fuente: Hoja de Resumen de Planillas. Elaboración: Fernando Cuadros Luque). 


\section{CONCLUSIONES}

1. La «idea-base» de un mercado global con una demanda de bienes y servicios cambiante y diversa que impone la flexibilización resulta altamente discutible, en la medida que se ha generalizado sin admitir particularidades por países, sistemas productivos o sectores económicos. En atención a ello, consideramos más plausible entender la flexibilidad como una estrategia empresarial diseñada para enfrentar la incertidumbre del mercado y de la tecnología a través de un mayor control del factor trabajo.

2. La flexibilidad se ha materializado en una «desrregulación» de la norma laboral. Ello, desde la perspectiva jurídica, conduce a la desnaturalización del contrato de trabajo, toda vez que permite al empleador trasladar hacia el trabajador los riesgos (incertidumbres) de su actividad empresarial, pese a que resulta esencial a dicho tipo de contrato la ajenidad en los riesgos para el trabajador.

3. Una primera diferencia que hemos identificado entre los procesos de flexibilización laboral llevados a cabo en España y Perú es que mientras el primero cuenta con la EEE como marco político de referencia, dicho marco político es inexistente en el caso peruano. En nuestra opinión, esta ausencia impide que la flexibilidad laboral sea objeto de un debate político y social serio y que se inserte como una medida coherente con otras políticas de índole social, educativa, fiscal y empresarial.

4. Un segundo aspecto diferenciador tiene que ver con la regulación de la contratación individual. Y es que aun cuando España y Perú comparten un mercado dual y precarizado - como efecto de la flexibilización-, el mayor problema en el caso peruano sigue siendo la informalidad laboral. Ello debido a que, con miras a combatirla, se ha creado un régimen diferenciado para los trabajadores de las micro y pequeñas empresas que supone - a grandes rasgos - la reducción generalizada de los derechos y beneficios laborales de aproximadamente el $76 \%$ de los asalariados privados.

5. Finalmente, las diferencias entre los modelos sindicales de España - centralizado, con vasta tradición en el diálogo social y amplia cobertura de la negociación - y de Perú —atomizado, con diálogo social incipiente y estrecha cobertura de la negociacióngeneran que la potencialidad de la negociación colectiva en el primero sea sustancialmente mayor que en el segundo.

6. En suma, pese a tratarse de un discurso generalizado, la implementación y los efectos de la flexibilidad laboral varían 
en cada Estado, dependiendo de un conjunto de características propias como son su cultura y tradición política, la estructura de su sistema productivo y la correlación de fuerzas entre sus actores sociales, tal como ha quedado en evidencia al comparar los casos de España y Perú.

Recibido: 9/01/2012

Aprobado: 16/03/2012 